\title{
What is the financial risk in GP commissioning?
}

\author{
'Nought from the Greeks towards me \\ hath sped well. \\ So now I find that ancient proverb true, \\ Foes' gifts are no gifts: profit bring they \\ none.' (Sophocles, 496-406 BC, in Ajax)
}

The aim of GP commissioning is quite rightly aligned to the wider issues surrounding cost containment in health care. Of necessity, this implies a budget and a list of items to be purchased. Implicit in the language of purchasing is the assumption of a high degree of control on behalf of the purchaser. Control implies predictability and direct ability to influence volatility. Does this implied level of direct control hold true in health care?

To understand the financial risk implied by GP commissioning, therefore, firstly implies an understanding of the volatility associated with healthcare costs. Low volatility means low risk and ease of forecasting, while high volatility means high risk and uncertain forecasting of future demand.

Volatility is determined by two factors. First, simple random variation around an average and, second, by additional volatility due to the linkage between the weather (changes in temperature, pressure, humidity, rainfall, and weather patterns), the environment (viruses, epidemics, pollution, water hardness/softness, background radon levels, and any other variable with a potential effect on health), and the expression of illness and disease in their widest sense.

Simple random variation is largely set Poisson statistics, which describe the range of outcomes around an average for whole number events; that is, 107 admissions or 223 outpatient attendances. By definition the standard deviation (a measure of volatility) of a Poisson distribution is equal to the square root of the mean. Therefore the standard deviation associated with an expected average of 100 admissions is $10( \pm 10 \%)$. At the level of a single GP practice there are very few acute events (with a unique cost) occurring at a frequency of 100 . Hence, while there is some offset between the 'overs' and 'unders' in numbers, the net risk arising from simple randomness is still high. This number-based risk is then magnified by the cost of the admission or attendance. Hence, one study has estimated that all events costing more than $£ 3000$ need to be excluded from a typical acute healthcare budget to achieve the point of minimum possible financial risk. ${ }^{1}$

Research also shows ${ }^{2}$ that the additional volatility arising from the environment leads to the actual uncertainty for elective and emergency admission being around twoand three-times higher respectively than due to simple randomness. As both simple statistical and environment-based volatility are not under the direct control of a GP, the respective costs incurred against the fixed budget is largely controlled by statistical and environmental chance-based events. The actual spend is therefore subject to a high degree of chance and some GPs make a profit and others a loss due to chance alone. ${ }^{1-5}$

The only way to counteract this is to reduce the long-term average total cost to something around three to four standard deviations worth of simple random-based variation below the funded (or average expected) cost. $^{5}$ Some tricky issues therefore exist around what to do with surpluses and the need to retain a surplus to cover possible losses in future years, and how to separate chance variation from higher costs due to 'poor performance'. 3,4

The next layer of complexity, or more correctly local bias, in costs and funding arises from the formula used to calculate the budget and the ability of acute trusts to exploit ambiguity in the healthcare resource group tariff (and the belated catch-up by the Department of Health to close these holes) leading to higher costs in one location than another.

All formula, no matter how seemingly sophisticated and comprehensive, contain hidden assumptions and flaws which result in a degree of relative over- and under- funding especially in those populations that deviate from the national 'average'; such as high affluence, deprivation, or ethnic composition. All formulae break down at the extremes. For example, the national weightings applied to the GMS-PMS formula contains seven age bands $(0-4$, $5-14,15-44,45-64,65-74,75-84$ and $\geq 85$ years, especially important for the age bands above the 65 years), and has the hidden assumption that local deviation from the national average population age profile within each age band has no influence on the calculated total weighting at practice level. Another example is that the proportion of privately insured individuals are not directly measured but inferred from other measures and that the proportion of students (who consume far lower levels of elective and outpatient care than their nonstudent counterparts), may not be accounted for.

Succeeding each iteration of the formulas different purchasers suddenly experience what may sometimes be large shifts in their baseline budget. In theory the statistical likelihood of each formula to over- and under-fund is available to the model designers; however, for somewhat obvious reasons this information is never released for wider discussion. Some practices will therefore have to make a degree of savings to compensate for whatever degree of local under-funding arises from the formula while others inherit a natural propensity to a surplus.

However, by far the greatest limitation of all formula, is the hidden assumption that all healthcare demand solely arises from person-based or population-based characteristics. Therefore, no formula devised to date contains any allowance for the range of regional weather patterns or the granularity associated with infectious outbreaks. These factors alone have the potential to introduce a level of significant bias into the issue of 'fair' funding.

The final issue is that of the expected future trajectory in healthcare funding versus the expected trajectory in cost 
pressures. $^{6-7}$ After some 18 years of grappling with forecasting healthcare demand, the author has concluded that the ageing population has very little to do with growth in costs; a view which is also shared by economists. ${ }^{6}$ Instead the observed trends appear to follow complex patterns which include step-changes and what may otherwise be called long-term cycles all mixed in with other aspects of care which follow more recognisable linear growth trajectories. Unexpected growth in future demand appears to be the norm rather than the exception. To make matters worse, no one at the department appears to be prepared to admit that this is the case and hence there is very little research into this poorly understood area which could then enlighten us to the future challenges.

In conclusion, this essay has been deliberately written at a non-technical level to attract the widest audience of GPs into the necessary debate required before GP commissioning is implemented. Ultimately, there is nothing to be gained from rushing headlong into what seemed like a good idea to someone else. Recent comments that commissioners were only worth a score of 3 out of 10 may actually be the 'red flag' letting us all know that commissioning in the real world is obfuscated by an, as yet unacknowledged, high background level of uncontrollable variation and hence, risk. To suggest otherwise is to imply that GPs have the power to control the weather and other natural phenomena - no doubt flattering, but sadly this remains untrue. To change one set of commissioners for another (including the private sector) will not solve these issues which will emerge from the woodwork once again as funding begins to be squeezed.

This is not an argument against the need to change the way health and social care is delivered but is a suggestion that a different set of commissioners may not be the best solution to a complex and poorly acknowledged problem.

Further reading giving relatively easy to understand discussion on the role of volatility in healthcare demand and financial risk calculation is available from: http://www.hcaf.biz/financialrisk.html

\section{Rodney $\mathbf{P}$ Jones}

\section{Competing interests}

The author provides healthcare actuarial and forecasting services.

\section{REFERENCES}

1. Jones R. Financial risk in practice based commissioning. British Journal of Healthcare Management 2008; 14(5): 199-204.

2. Jones R. Emergency admissions and financial risk. British Journal of Healthcare Management 2009; 15(7): 344-350.

3. Jones R. Financial risk at the PCT/PBC Interface. British Journal of Healthcare Management 2008; 14(7): 288-293.

4. Jones R. The actuarial basis for financial risk in practice-based commissioning and implications to managing budgets. Prim Health Care Res Dev 2009; 10(3): 245-253.

5. Jones R. Financial risk in health purchasing: risk pools. British Journal of Healthcare Management 2008; 14(6): 240-245.

6. Jones $\mathrm{R}$. The nature of health care costs and financial risk in commissioning. British Journal of Healthcare Management 2010; 16: (in press).

7. Jones R. Myths of ideal hospital size. Med J Aust 2010; 193: (in press).

DOI: 10.3399/bjgp10X515575 